\title{
ANÁLISE DO FILTRO DE KALMAN ESTENDIDO PARA DETERMINAÇÃO DE ÓRBITA A BORDO CONSIDERANDO ARCOS CURTOS
}

\author{
ANA PAULA M. CHIARADIA ${ }^{1}$, HÉLIO. K. KUGA², BRUNA Y. P. L MASAGO ${ }^{2}$ \\ 1. Grupo de Dinâmica Orbital e Planetologia - UNESP - Campus de Guaratinguetá -Av. Ariberto \\ Pereira da Cunha, 333 - Pedregulho - Guaratinguetá - SP - CEP: 12513-485 \\ e-mails: anachiaradia@feg.unesp.br
}
2. Instituto Nacional de Pesquisas Espaciais, Divisão de Mecânica Espacial e Controle Av. dos Astronauta, 1758 - São José dos Campos - SP - CEP12.223-010
E-mails: brunamasago@gmail.com; hkk@dem.inpe.br

\begin{abstract}
This work is concerned with orbit determination using GPS signals. An especial case of truncated arcs assuming that GPS receiver data, which is available only when the satellite carrying the receiver flies over a ground tracking station, is the main issue. To analyse the behaviour of an Extended Kalman filter (EKF) in real time satellite orbit determination using short arcs of data, the algorithm developed by Chiaradia et.al. (2013) is used. This algorithm has been formerly qualified using the raw single frequency pseudorange GPS measurements of the Topex/Poseidon (T/P) satellite as observations by the Kalman Filter, and for this reason, is used as reference in this work. However, these real data are truncated as if they had been collected by the tracking and control station of INPE in Cuiabá, Brazil. That is, the data are obtained only when the satellite T/P is in the viewing area of the Cuiaba Brazilian station. The behaviour of the Kalman filter is analysed under such premises. The results of research are presented showing the degradation of performance when compared to the full arc orbit determination.
\end{abstract}

Keywords— Kalman filter, short arcs, GPS, orbital dynamics.

Resumo— Este trabalho trata-se de determinação de órbita usando sinais GPS. Um caso especial de arcos truncados é a questão principal, isto é, será suposto que os dados do receptor GPS somente estarão disponíveis quando o satélite com um receptor GPS abordo estiver sobrevoando uma estação terrestre de rastreamento. Para analisar o comportamento de um filtro de Kalman estendido (EKF) na determinação de órbita de satélites em tempo real usando arcos curtos de dados é usado o algoritmo desenvolvido por Chiaradia et. al. (2013). Este algoritmo foi anteriormente qualificado usando as medidas GPS de pseudodistância real em uma única frequência do satélite Topex/Poseidon (T/P) como dados de observações do filtro Kalman e, por esta razão, é usado como referência neste trabalho. No entanto, estes dados reais são truncados, como se eles tinham sido coletados pela estação do Centro de Controle e Rastreamento do INPE em Cuiabá, Brasil. Ou seja, os dados são obtidos somente quando o satélite T/P está na área de visibilidade da estação brasileira de Cuiabá. O comportamento do filtro de Kalman é analisado. Os resultados da pesquisa são apresentados mostrando a degradação do desempenho quando comparado com a determinação da órbita de arco completo.

Palavras-chave— Filtro de Kalman, arcos curtos, GPS, dinâmica Orbital.

\section{Introdução}

Apesar do GPS fornece precisão em torno de centímetros e as estações terrestres de controle fornecem precisão padrão cerca de cem metros, o Instituto Nacional de Pesquisas Espaciais (INPE) ainda usa estações terrestres de controle para determinar e controlar a órbita de seus satélites artificiais, como o SCD1 (Kuga e Kondapalli, 1993; Orlando et. al, 1997), SCD2 (Kuga. Orlando e Lopes, 1999; Orlando e Kuga, 2007), do CBERS-1 (Kuga e Orlando, 2001) e CBERS-2 (Orlando, Kuga e Tominaga, 2004).

O sistema de rastreamento terrestre do INPE, desenvolvido para controlar os satélites e sua estrutura funcional, é chamado pelo Centro de Rastreio e Controle de satélite (CRC). Este centro é composto de Centro de Controle de Satélites (CCS), localizado em São José dos Campos-Brasil e duas estações terrenas de rastreio de Cuiabá $\left(23^{\circ} 12^{\prime} \mathrm{S}\right.$, $\left.45^{\circ} 51^{\prime} \mathrm{W}\right)$ e de Alcântara ( $\left.2^{\circ} 20^{\prime} \mathrm{S}, 44^{\circ} 24^{\prime} \mathrm{W}\right)$ (Kuga e Orlando, 2001).

Com a finalidade de testar a possibilidade de utilizar o algoritmo desenvolvido por Chiaradia et. al. (2013) para determinar órbitas de satélites artificiais pelo INPE, será simulado o rastreamento do satélite T/P pela estação de rastreamento e controle localizada em Cuiabá. O objetivo é analisar a eficiência deste algoritmo para determinar a órbita com arcos curtos. Isto é, os dados do receptor GPS do T/P somente estarão disponíveis quando o satélite estiver visível pela estação terrestre de rastreamento de Cuiabá.

\section{Descrição do algoritmo para determinação de órbita}

Neste trabalho, o algoritmo desenvolvido por Chiaradia et. al. (2013) é usado para determinar a órbita do satélite a bordo, em tempo real usando o sistema GPS. Este algoritmo utiliza como método de estimação o Filtro de Kalman Estendido (EKF). Este estimador foi utilizado por ser robusto, fácil imple- 
mentação, aplicação em tempo real, recursivo e sequencial, isto é, não é necessário armazenamento de observações podendo estas serem processadas à medida que vão sendo coletadas e a convergência é obtida à medida que uma certa quantidade de observações é processada. A seguir são descritas as equações do Filtro de Kalman de acordo com Gelb et al. (1974).

Seja a dinâmica, contínua no tempo, modelada como uma função não-linear do estado na seguinte forma:

$$
\dot{\mathbf{x}}(t)=\mathbf{f}(t, \mathbf{x}(t))+\mathbf{B}(t) \mathbf{w}(t)
$$

em que $\boldsymbol{x}$ é o vetor de estado, que neste trabalho é dado por:

$$
\mathbf{x}=(\mathbf{r}, \mathbf{v}, \mathbf{b})^{T}
$$

em que $\mathbf{r}=(\mathrm{x}, \mathrm{y}, \mathrm{z})^{\mathrm{T}}$ e $\mathbf{v}=(\dot{x}, \dot{y}, \dot{z})^{T}$ são os vetores posição e velocidade do veículo espacial; $\mathbf{b}=\left(b_{0}, b_{1}, b_{2}\right)^{T}$, onde $b_{0}, b_{1}, b_{2}$, são a tendência (bias), deriva (drift) e taxa de deriva (drift rate) do relógio do receptor GPS, respectivamente; $t$ é o instante de observação; $\boldsymbol{f}$ é uma função vetorial do estado e do tempo de dimensão $n ; \boldsymbol{B}$ é uma matriz $n \times \mathrm{x} r$ contínua no tempo; e $\boldsymbol{w}$ é o processo gaussiano branco de média nula e covariância $\boldsymbol{Q}$, isto é:

$$
\begin{aligned}
& E[\mathbf{w}(t)]=0 \\
& E\left[\mathbf{w}(t) \mathbf{w}^{T}(\tau)\right]=\mathbf{Q}(t) \delta(t-\tau)
\end{aligned}
$$

ou seja, $\mathbf{w}(t)=N(0, \mathbf{Q}(t))$, onde $E[$.] representa o operador esperança, $\boldsymbol{Q}$ é uma matriz $n x n$ de densidade espectral do ruído da dinâmica semi-definida positiva e $\delta$ é a função Delta de Dirac.

Neste trabalho, as coordenadas de posição e de velocidade estão em metros e metros/segundos, respectivamente. Todas as coordenadas estão referenciadas no sistema inercial ToD (True of Date).

Sejam as observações, discretas no tempo, modeladas como uma função não-linear do estado, na seguinte forma:

$$
\mathbf{y}(k)=\mathbf{h}(k, \mathbf{x}(k))+\mathbf{v}(k)
$$

em que $\boldsymbol{y}$ é o vetor de $m$ observações, $\boldsymbol{h}$ é uma função vetorial m-dimensional do estado e do instante $k, v$ é uma seqüência gaussiana branca de média nula e covariância $\boldsymbol{R}$, isto é:

$$
\begin{aligned}
& E[\mathbf{v}(k)]=0 \\
& E\left[\mathbf{v}(k) \mathbf{v}^{T}(j)\right]=\mathbf{R}(k) \delta_{k j}
\end{aligned}
$$

ou seja, $\mathbf{v}_{k}=N\left(0, \mathbf{R}_{k}\right)$, onde $\boldsymbol{R}$ é a matriz de covariância $m \times m$ dos erros de observação definida positiva.

O ruído branco $\boldsymbol{w}$ é não-correlacionado, tanto com o estado inicial $\boldsymbol{x}$ quanto com a seqüência $\boldsymbol{v}$ e a seqüência $v$ é não-correlacionada com o estado inicial, portanto:

$$
\begin{aligned}
& E\left[\mathbf{w}\left(t_{k}\right) \mathbf{v}^{T}(k)\right]=0 \\
& E\left[\mathbf{x}\left(t_{k}\right) \mathbf{v}^{T}(k)\right]=0 \\
& E\left[\mathbf{x}\left(t_{0}\right) \mathbf{w}(t)\right]=0
\end{aligned}
$$

A equação da dinâmica é propagada a partir da condição inicial do estado $\boldsymbol{x}_{0}$. Como este valor não pode ser conhecido precisamente a priori, é modelado como um vetor aleatório que é normalmente distribuído. A descrição de $\boldsymbol{x}\left(t_{0}\right)$ é completamente especificada pela média $\hat{\mathbf{x}}_{0}$ e covariância $\boldsymbol{P}_{0}$. Então, as condições iniciais são também gaussianas e nãotendenciosas:

$$
\begin{aligned}
& E\left[\mathbf{x}\left(t_{0}\right)\right]=\hat{\mathbf{x}}_{0} \\
& E\left[\left(\mathbf{x}\left(t_{0}\right)-\hat{\mathbf{x}}_{0}\right)\left(\mathbf{x}\left(t_{0}\right)-\hat{\mathbf{x}}_{0}\right)^{T}\right]=\mathbf{P}_{0}
\end{aligned}
$$

em que $t_{0}$ é o instante inicial, $\boldsymbol{P}$ é a matriz de covariância do erro do estado e $\hat{\boldsymbol{x}}$ representa a melhor estimativa do estado no instante considerado.

A estimativa do estado e da matriz de covariância de seu erro, no próximo instante de observação, são calculadas com auxílio do modelo da dinâmica do sistema. Elas são propagadas até os instantes de observação, mas antes da medida ser incorporada. As equações de propagação do estado $\overline{\boldsymbol{x}}$, da covariância $\overline{\mathbf{P}}$ e da matriz de transição do estado $\boldsymbol{\Phi}$ são dadas por:

$$
\begin{aligned}
& \dot{\overline{\mathbf{x}}}(t)=\mathbf{f}(t, \overline{\mathbf{x}}(t)) \\
& \dot{\boldsymbol{\Phi}}(t)=\mathbf{F}(t, \overline{\mathbf{x}}(t)) \boldsymbol{\Phi}(t) \\
& \overline{\mathbf{P}}(k+1)=\boldsymbol{\Phi}(k) \hat{\mathbf{P}}(k) \boldsymbol{\Phi}^{T}(k)+ \\
& \int_{k}^{k+1} \boldsymbol{\Phi}(k, \tau) \mathbf{B}(\tau) \mathbf{Q}(\tau) \mathbf{B}^{T}(\tau) \boldsymbol{\Phi}(k, \tau) d \tau
\end{aligned}
$$

em que $\overline{\mathbf{x}}\left(t_{0}\right)=\hat{\mathbf{x}}\left(t_{0}\right), \boldsymbol{\Phi}\left(t_{0}\right) \equiv \boldsymbol{I}, \boldsymbol{I}$ é a matriz identidade e $\mathbf{F}=\partial \mathbf{f}(\mathbf{x}, \mathrm{t}) / \partial \mathbf{x}$. Na prática as Eqs. (13) e (14) devem ser integradas simultaneamente, no mesmo conjunto de equações diferenciais para assegurar que as derivadas parciais estão sendo calculadas na trajetória de referência $\overline{\boldsymbol{x}}$.

A matriz $\boldsymbol{\Phi}$ descreve a evolução do erro entre o estado de referência e o estado verdadeiro entre instantes diferentes. A matriz $\boldsymbol{P}$ é a medida da exatidão do erro de estado. No final do processo, $\overline{\mathbf{x}}$ e $\overline{\mathbf{P}}$ são obtidos e são chamados de estado e covariância atualizados, respectivamente.

Porém, neste trabalho, o estado é propagado utilizando um integrador numérico. A matriz de transição para propagar a matriz de covariância $\boldsymbol{P}$ é dada por:

$$
\begin{gathered}
\boldsymbol{\Phi}\left(t, t_{0}\right)=\left(\begin{array}{lll}
\boldsymbol{\Phi}_{11} & \boldsymbol{\Phi}_{12} & \boldsymbol{\Phi}_{13} \\
\boldsymbol{\Phi}_{21} & \boldsymbol{\Phi}_{22} & \boldsymbol{\Phi}_{23} \\
\boldsymbol{\Phi}_{31} & \boldsymbol{\Phi}_{32} & \boldsymbol{\Phi}_{33}
\end{array}\right)= \\
\left(\begin{array}{lll}
\frac{\partial \mathbf{r}}{\partial \mathbf{r}_{0}} & \frac{\partial \mathbf{r}}{\partial \mathbf{v}_{0}} & \frac{\partial \mathbf{r}}{\partial \mathbf{b}_{0}} \\
\frac{\partial \mathbf{v}}{\partial \mathbf{r}_{0}} & \frac{\partial \mathbf{v}}{\partial \mathbf{v}_{0}} & \frac{\partial \mathbf{v}}{\partial \mathbf{b}_{0}} \\
\frac{\partial \mathbf{b}}{\partial \mathbf{r}_{0}} & \frac{\partial \mathbf{b}}{\partial \mathbf{v}_{0}} & \frac{\partial \mathbf{b}}{\partial \mathbf{b}_{0}}
\end{array}\right)
\end{gathered}
$$

em que as submatrizes $\boldsymbol{\Phi}_{11}, \boldsymbol{\Phi}_{12}, \boldsymbol{\Phi}_{21}$, e $\boldsymbol{\Phi}_{22}$ são calculadas de Chiaradia et. (2012); as submatrizes $\boldsymbol{\Phi}_{13}$, $\Phi_{23}, \Phi_{31}$, e $\boldsymbol{\Phi}_{32}$ são nulas; e a submatriz $\boldsymbol{\Phi}_{33}$ é a matriz de transição, correspondente à tendência, deriva e taxa de deriva do relógio do receptor GPS, dada por: 


$$
\boldsymbol{\Phi}_{33}\left(t, t_{0}\right)=\left[\begin{array}{lll}
1 & 0 & 0 \\
0 & 1 & 0 \\
0 & 0 & 1
\end{array}\right]
$$

As estimativas do estado e da matriz de covariância de seu erro, propagadas para o instante da observação atual, são atualizadas com base no processamento das observações. As equações de atualização do estado $\hat{\boldsymbol{x}}$, da covariância $\hat{\mathbf{P}}$ e do ganho de Kalman $\mathbf{K}$ são dadas por:

$$
\begin{aligned}
& \mathbf{K}(k+1)=\overline{\mathbf{P}}(k+1) \mathbf{H}^{T}(k+1, \overline{\mathbf{x}}(k+1)) \\
& \left(\mathbf{H}(k+1, \overline{\mathbf{x}}(k+1)) \overline{\mathbf{P}}(k+1) \mathbf{H}^{T}(k+1, \overline{\mathbf{x}}(k+1))+\mathbf{R}(k+1)\right)^{-1} \\
& \hat{\mathbf{P}}(k+1)=(\mathbf{I}-\mathbf{K}(k+1) \mathbf{H}(k+1, \overline{\mathbf{x}}(k+1)) \overline{\mathbf{P}}(k+1) \\
& \hat{\mathbf{x}}(k+1)=\overline{\mathbf{x}}(k+1)+\mathbf{K}(k+1)(\mathbf{y}(k+1)-\mathbf{h}(k+1, \overline{\mathbf{x}}(k+1))
\end{aligned}
$$

em que $\boldsymbol{H}$ é a matriz de sensibilidade dada pelas derivadas parciais de $\boldsymbol{h}$ em relação a $\boldsymbol{x}$ avaliada ao longo da trajetória nominal:

$$
\mathbf{H}[k+1, \overline{\mathbf{x}}(k+1)]=\left[\frac{\partial \mathbf{h}(k+1, \mathbf{x})}{\partial \mathbf{x}}\right]_{x=\bar{x}(k+1)}
$$

As Eqs. (18) a (20) podem ser usadas para processar a medida, sequencialmente, de modo que a inversão de matriz na Eq. (18) é transformada na inversão de um escalar. As medidas devem ser nãocorrelacionadas e, neste caso, a matriz $\boldsymbol{R}$ é diagonal.

$\mathrm{O}$ resíduo da medida e a matriz de sensibilidade são encontrados através da equação de medida calculada pela equação dada:

$$
\rho_{c}=\rho+c\left[\Delta_{G P S}(t)-\Delta_{u}(t)\right]+\Delta_{I O N}+\Delta_{T R O}+\mathcal{E}=c \tau
$$

Usando a pseudo-distância, a matriz de sensibilidade é dada por:

$$
\mathbf{H}\left[\frac{\left(x_{G P \bar{S}}\right)}{\rho} \frac{\left(y_{G P \bar{S}}\right)}{\rho} \frac{\left(z_{G P S^{2}}\right)}{\rho} 000001 \Delta \Delta^{2}\right]
$$

em que $\Delta T$ é o tempo acumulado desde a primeira medida. O resíduo da medida é:

$$
\Delta \boldsymbol{\rho}(k)=\mathbf{y}(k)-\boldsymbol{\rho}_{c}(\mathbf{x}(t), t)
$$

em que $\boldsymbol{y}$ é a medida observada e $\boldsymbol{\rho}_{\boldsymbol{c}}$ é a medida calculada pela Eq. (22). A matriz $\boldsymbol{R}$ é, basicamente, uma matriz do peso da medida, que expressa a magnitude dos erros de medida.

O modelo simplificado de força foi adotado considerando apenas as forças devido ao campo gravita-cional terrestre. Os coeficientes harmônicos considerados foram na ordem e grau 10 do modelo JGM-2, de acordo com estudos desenvolvidos em Chiaradia et al. (2003), sem sobrecarregar o tempo de processamento. A aceleração e as matrizes das derivadas parciais são calculadas através da relação de recorrência, de acordo com Pines (1973), em coordenadas terrestres fixas. A transformação de coordenadas do sistema inercial ToD (True of Date) para PEF leva em conta a rotação sideral da Terra, porém o movimento polar é negligenciado.

A integração da equação de movimento do satélite foi realizada usando o integrador numérico Runge-Kutta de passo fixo de quarta ordem (RK4).
Foi implementado sem qualquer mecanismo de ajuste de passo ou controle de erro. Não foi necessário utilizar qualquer procedimento de inicialização e o tamanho do passo é muito fácil de ser alterado.

A matriz de covariância dos erros do estado foi propagada através da matriz de transição calculada de maneira otimizada. (Chiaradia et al. 2012).

As medidas GPS de pseudodistância em uma única frequência foram utilizadas como medidas de observação dada pela Eq. (22). Estas foram corrigidas com relação ao atraso dos relógios dos satélites GPS e do receptor. O efeito ionosférico foi considerado nas medidas através do modelo de dupla frequência, somente para avaliação do impacto na precisão.

Para analisar o algoritmo desenvolvido, Chiaradia et. al. (2013) utilizou os dados do T/P, porque carregava um receptor de dupla frequência GPS a bordo experimentalmente para testar a capacidade do GPS para fornecer a determinação da órbita precisa (POD). A órbita do satélite foi estimada usando o algoritmo desenvolvido com uma boa precisão e mínimo custo computacional. A precisão de posição com SA ou sem foi estimada de 15 a $20 \mathrm{~m}$ com desvio padrão de 6 a $10 \mathrm{~m}$. E a precisão da velocidade foi estimada de 0,014 a 0,018 $\mathrm{m} / \mathrm{s}$, com desvio padrão de 0,006 a $0,009 \mathrm{~m} / \mathrm{s}$.

\section{Simulações dos arcos curtos}

Como o algoritmo usado para determinar a órbita de um satélite artificial foi qualificado usando as medições GPS de pseudodistância em única frequência do satélite T/P como observações, os mesmos dados são utilizados como referência neste trabalho. No entanto, estes dados reais são simulados como se eles tivessem sido coletados pela estação de rastreamento e controle do INPE em Cuiabá, Brasil. Ou seja, os dados são obtidos somente quando o satélite T/P está na área de visualização da estação brasileira de Cuiabá.

Assim, como o raio de rastreio da estação terrena de Cuiabá é 4, 300km, o T/P está visível para Cuiabá durante arcos curtos, como mostra a Figura 2. O período orbital do T/P é de $112 \mathrm{~min}$.

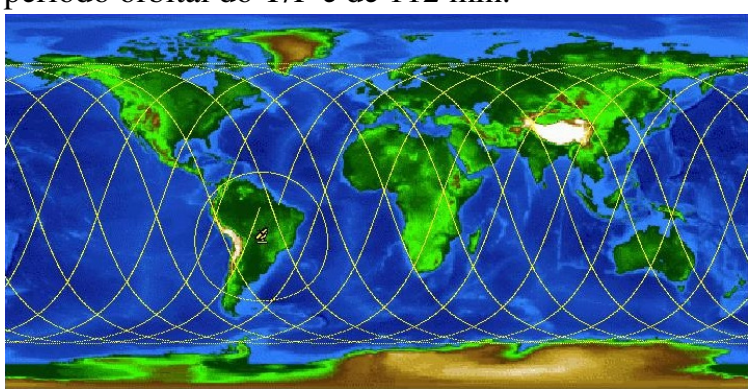

Figura 2. Simulação da órbita T/P

Os dados de observação do T/P do dia 18/11/1993 foram monitorados pela estação terrestre de Cuiabá. Neste dia, o SA estava desativada. O período de visibilidade do T/P é mostrado na Tabela 
1 para o dia 18/11/1993. Neste, há quatro arcos curtos visíveis.

Tabela 1. Período dos Arcos Curtos.

\begin{tabular}{|c|c|c|}
\hline Arcos Curtos & $\begin{array}{c}\text { Início } \\
\text { (hh mm ss.s) }\end{array}$ & $\begin{array}{c}\text { Fim } \\
\text { (hh mm ss.s) }\end{array}$ \\
\hline 1 & 014359.0 & 015554.0 \\
\hline 2 & 120620.0 & 122429.0 \\
\hline 3 & 140305.0 & 141927.0 \\
\hline 4 & 221346.0 & 222827.0 \\
\hline
\end{tabular}

\section{Análise dos dados}

No algoritmo de Chiaradia et. al (2013) foram utilizados os seguintes arquivos que também são utilizados neste trabalho: os arquivos de observação de T/P que transporta o as medidas de pseudodistância e da fase da portadora em duas frequências em intervalos de tempo GPS de 10 segundos de transmissão e são fornecidos pelo Jet Propulsion Laboratory (JPL) no formato RINEX; os arquivos de efemérides de órbita precisa T/P (POE) que são gerados pelo JPL; e a mensagem de navegação do GPS transmitida em formato RINEX, fornecido pelo Crystal Dynamics Data Information System (CDDIS) do Goddard Space Flight Centre (GSFC).

As coordenadas estimadas da posição e velocidade são comparadas com os arquivos de referência $\mathrm{POE}$ do T/P. O arquivo POE do satélite T/P fornece as coordenadas da posição do T/P com precisão menor do que $15 \mathrm{~cm}$, e fornecem dados em um intervalo de tempo de um minuto UTC no sistema ToD. Entretanto, no processo de determinação de órbita o estado é estimado em intervalos de 30 segundos no tempo UTC com correção do tempo de emissão e recepção. Logo, os instantes dos dados para comparação não coincidem. Então, foi necessário interpolar os dados de referência. Para isto, foi utilizada a subrotina de interpolação Polint (Press et al., 1987). Com este enfoque, o erro médio de interpolação é de $0,068 \mathrm{~m} \mathrm{e}$ $2,5 \times 10^{-4} \mathrm{~m} / \mathrm{s}$ para posição e velocidade, respectivamente.

Para o dia 18/11/1993, foi usado um arquivo de observação completamente bruto; isto é, medidas negativas. Os mesmos parâmetros de entradas do filtro e as condições iniciais foram utilizados neste trabalho.

Em Chiaradia et. al (2013), a precisão de posição com o SA para o dia 18/11/1993 é de $15,5 \mathrm{~m}$, com desvio padrão de $6,8 \mathrm{~m}$. A precisão de velocidade é de 0,014 , com desvio padrão de $0,006 \mathrm{~m} / \mathrm{s}$, conforme mostrado na Tabela 2.

Os erros real em posição é dado por:

$$
\Delta \mathbf{r} \equiv\left[\sum_{i=1}^{3}\left(x_{i}-\hat{x}_{i}\right)^{2}\right]^{\frac{1}{2}}
$$

em que $x_{i}$ e $\hat{x}_{i}, i=1,2,3$, são as componentes de referência (ou real) e estimadas da posição do vetor de estado, respectivamente. $\mathrm{O}$ erro estimado em posição é dado por:

$$
\Delta \hat{\boldsymbol{r}}=\left[\sum_{i=1}^{3} \boldsymbol{P}_{i i}\right]^{\frac{1}{2}}
$$

em que $P_{i i}, i=1,2,3$, representam os valores dos elementos da diagonal da matriz de covariância dos erros na estimativa do vetor estado, correspondente aos elementos de posição. O erro real em velocidade é dado por:

$$
\Delta v \equiv\left[\sum_{i=4}^{6}\left(x_{i}-\hat{x}_{i}\right)^{2}\right]^{\frac{1}{2}}
$$

em que $x_{i}$ e $\hat{x}_{i}, i=4,5,6$, são as componentes de referência (ou real) e estimadas da velocidade do vetor de estado, respectivamente. O erro estimado em velocidade é dado por:

$$
\Delta \hat{\boldsymbol{v}}=\left[\sum_{i=4}^{6} \boldsymbol{P}_{i i}\right]^{\frac{1}{2}}
$$

em que $P_{i i}, i=4,5,6$, representam os valores dos elementos da diagonal da matriz de covariância dos erros na estimativa do vetor estado, correspondente aos elementos de velocidade. E o resíduo é dado por:

$$
\Delta \boldsymbol{\rho}=\boldsymbol{y}-\boldsymbol{\rho}_{c}
$$

em que $\boldsymbol{y}$ e $\boldsymbol{\rho}_{\boldsymbol{c}}$ são as medidas da pseudodistância observadas e calculadas, respectivamente.

Simulando os quatro arcos curtos utilizando o algoritmo nas mesmas condições, obtendo os seguintes dados. Na Figura 3 são apresentados os erros em posição real, estimado ao longo do dia inteiro com os quatros arcos curtos. Nas Figuras 4 a 7 são apresentados os erros em posição para cada arco curto. $\mathrm{Na}$ Figura 8 são apresentados os erros em velocidade real, estimado ao longo do dia inteiro com os quatros arcos curtos. Nas Figuras 9 a 12 são apresentados os erros em velocidade para cada arco curto.

Na Tabela 2 são apresentados os resultados obtidos em Chiaradia et. al. (2013) na primeira linha e os resultados para os quatros arcos curtos. Para todos os casos, são apresentados os erros em posição e velocidade e o resíduo com os seus respectivos desvios padrões, calculados de acordo com as equações (2) a (6).

\section{Conclusões}

Analisando os resultados obtidos, observou-se que somente o $2^{\circ}$ arco curto não obteve bom resultados tanto em posição quanto em velocidade. Os outros arcos os resultados foram obtidos dentro do valor 
esperado comparados com os valores obtidos para um dia completo, como em Chiaradia et. al. (2013).

Tabela 2. Resultados obtidos

\begin{tabular}{|c|c|c|c|}
\hline & $\Delta \mathbf{r}(\mathbf{m})$ & $\Delta \mathbf{v}(\mathbf{m})$ & Resíduo (m) \\
\hline $\begin{array}{c}\text { Ao longo } \\
\text { do dia }\end{array}$ & $15,5 \pm 6,8$ & $0,014 \pm 0,006$ & $0,027 \pm 13,2$ \\
\hline $\mathbf{1}^{\mathbf{o}}$ Arco & $31,2 \pm 1,4$ & $0,025 \pm 0,011$ & $-2,112 \pm 9,59$ \\
\hline $\mathbf{2}^{\mathbf{o}}$ Arco & $32,6 \pm 21,3$ & $0,14 \pm 0,1$ & $-1,640 \pm 87,6$ \\
\hline $\mathbf{3}^{\mathbf{o}}$ Arco & $10,1 \pm 5,7$ & $0,049 \pm 0,013$ & $2,112 \pm 10,9$ \\
\hline $\mathbf{4}^{\mathbf{o}}$ Arco & $34,5 \pm 6,3$ & $0,062 \pm 0,023$ & $1,564 \pm 16,4$ \\
\hline
\end{tabular}

\section{Agradecimentos}

Os autores agradecem o apoio recebido pela UNESP, INPE, FAPESP, CNPq e CAPES.

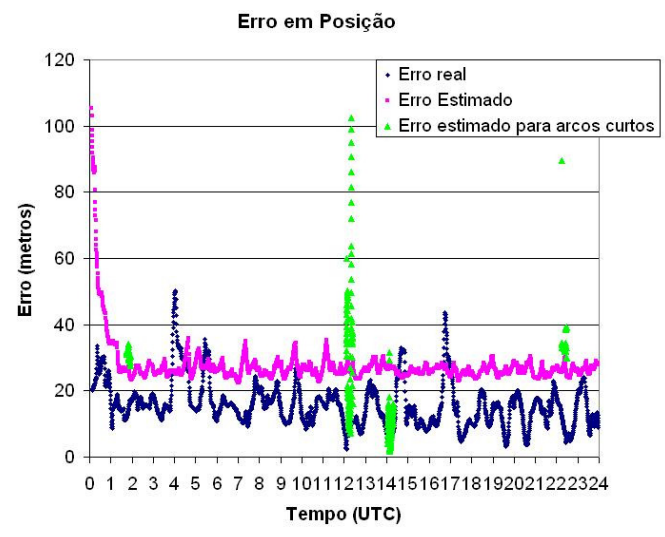

Figura 3. Erro em posição ao longo do dia.

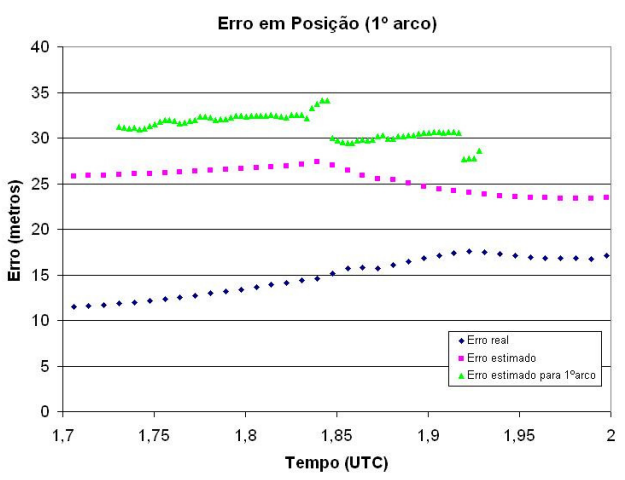

Figura 4. Erro em posição para o primeiro arco curto

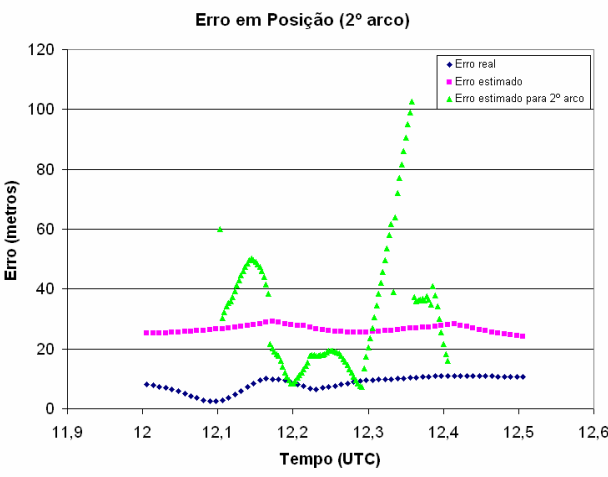

Figura 5. Erro em posição para o segundo arco curto

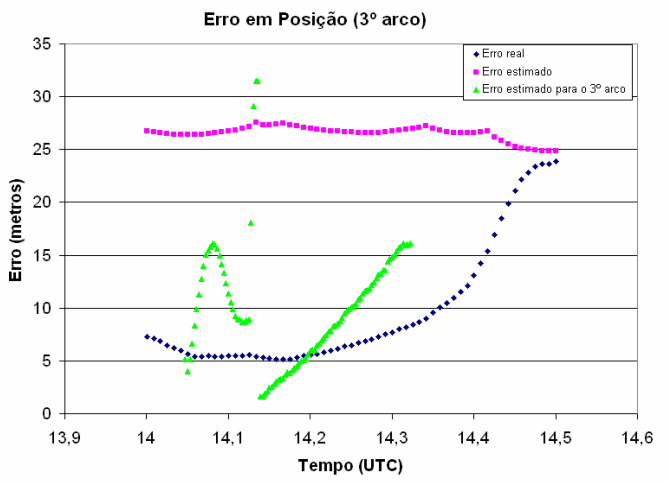

Figura 6. Erro em posição para o terceiro arco curto

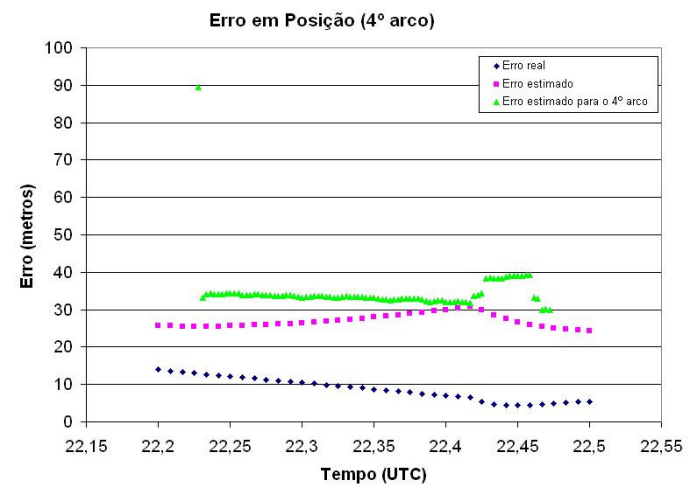

Figura 7. Erro em posição para o quarto arco curto

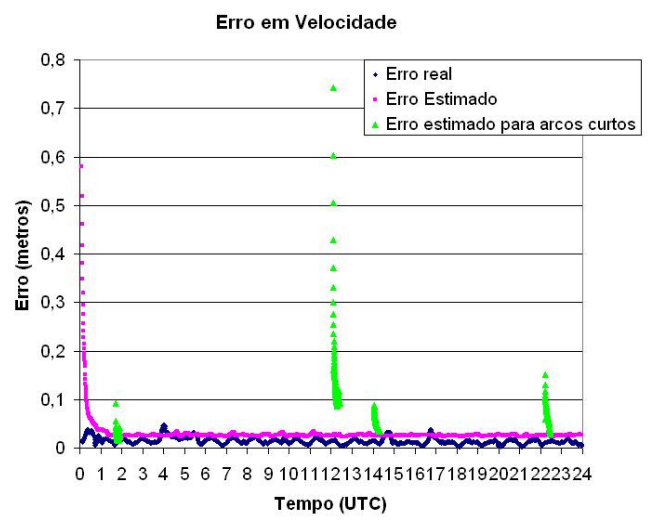

Figura 8. Erro em velocidade ao longo do dia 


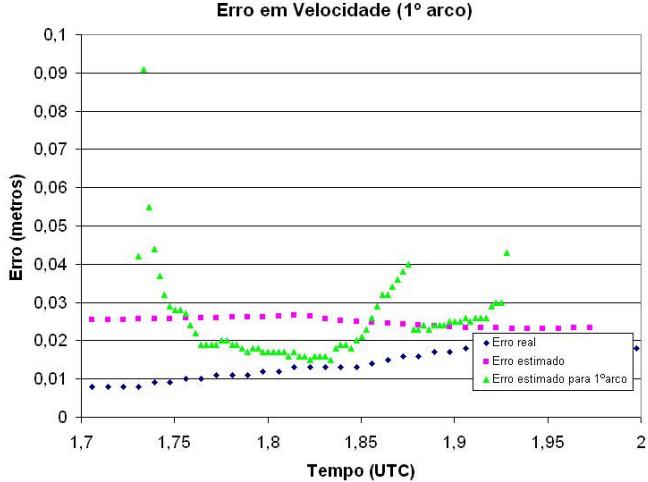

Figura 9. Erro em velocidade para o primeiro arco curto

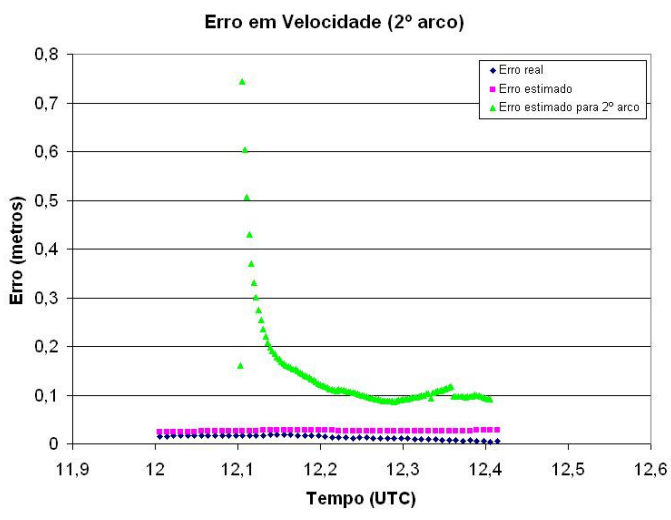

Figura 10. Erro em velocidade para o segundo arco curto

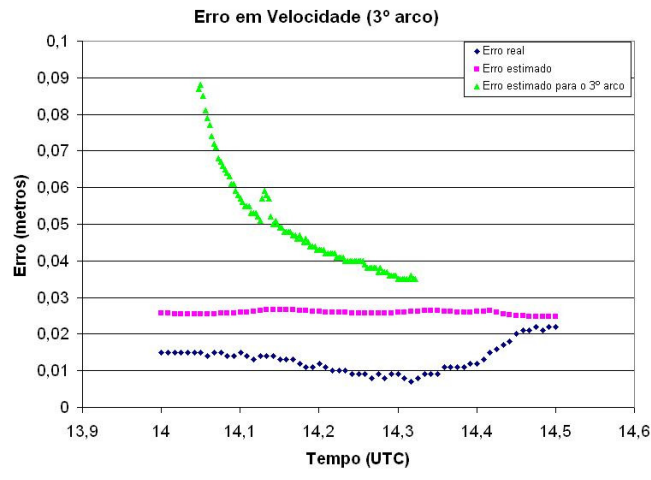

Figura 11. Erro em velocidade para o terceiro arco curto

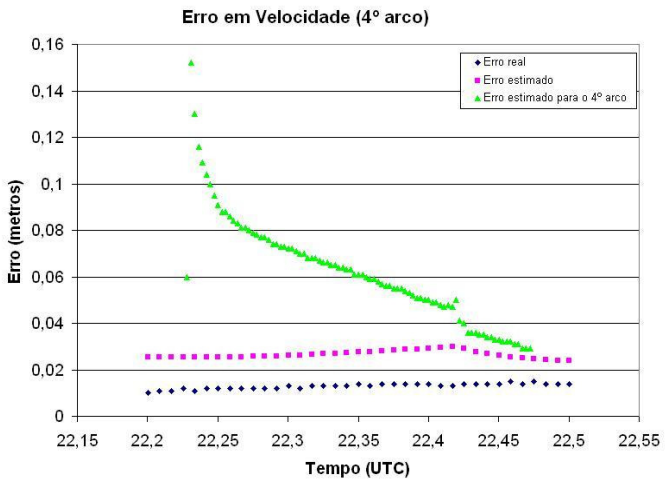

Figura 12. Erro em velocidade para o quarto arco curto

\section{Referências Bibliográficas}

Chiaradia, A. P. M. Kuga, H. K; Prado, A. F. B. A. Single Frequency GPS measurements in realtime artificial satellite orbit determination. ACTA ASTRONAUTICA. Vol. 53 Issue: 2 Pages: 123-133 Published: JUL, 2003. DOI: 10.1016/S0094-5765(02)00198-4

Chiaradia, A. P. M. Kuga, H. K; Prado, A. F. B. A. Comparison between Two Methods to Calculate the Transition Matrix of Orbit Motion. Mathematical Problems in Engineering (Print). v. 2012, p.1, - 12, 2012.

Chiaradia, A. P. M. Kuga, H. K; Prado, A. F. B. A. Onboard and real-time artificial satellite orbit determination using GPS Mathematical Problems in Engineering . Volume 2013, Article ID 530516, 8 páginas. Mathematical Problems in Engineering (Print), 2013. DOI: $10.1155 / 2013 / 530516$

Gelb, A.; Kasper Jr., J.F.; Nash Jr., R. A.; Price, C. F.; Sutherland Jr., A. A. Applied optimal estimation. Inglaterra: The M.I.T. Press, 1974. $374 p$.

INPE, Acesso online http://www.inpe.br/, no dia 03 de junho de 2013.

Kuga, H. K.; Kondapalli, R. R. "Satellite Orbit Determination: A First-Hand Experience with the First Brazilian Satellite SCD1", 44th. Congress of the IAF, Graz, Austria, October 1993.

Kuga, H. K.; Orlando, V. "Orbit Control of CBERS1 Satellite at INPE", 16th International Symposium on Spaceflight Dynamics, JPL/NASA, Pasadena, USA, 2001.

Kuga, H. K.; Orlando, V.; Lopes, R. V. F. "Flight Dynamics Operations During Leop for the INPE's Second Environmental Data Collecting Satellite SCD2", Revista Brasileira de Ciências Mecânicas, Vol. XXI, Special Issue, ISSN 01007386, 1999, pp. 339-344.

Orlando, V.; Kuga, H. K. "Os Satélites SCD1 e SCD2 da Missão Espacial Completa BrasileiraMECB", Capítulo 5 do livro "A Conquista do Espaço - Do Sputnik à Missão Centenário", 1a edição, São Paulo, SO, Editora Livraria da Física, v.1, p. 151-176, outubro de 2007.

Orlando, V.; Kuga, H. K.; Tominaga, J. "CBERS-2 LEOP Orbit Analysis",18th International Symposium on Spaceflight Dynamics, Munique, Alemanha, outubro de 2004, ISBN 929092859X, pp. 221-225.

Orlando, V.; Lopes, R. V. F.; Kuga, H. K. “INPE's Flight Dynamics Team Experience throughout Four Years of SCD1 In-Orbit Operations: Main Issues, Improvements and Trends", "ESA International Symposium on Spaceflight Dynamics", ESA, Darmstadt, Alemanha, junho, 1997. 
Pines, S. Uniform representation of the gravitational potential and its derivatives. AIAA Journal, 1973, v. 11, n. 11.

Press, H. W.; Flannery, B. P.; Teukolsky, S. A.;

Vetterling, W. T. Numerical Recipes.

Cambridge: University Press, 3. ed., 1987. 818p. 